\section{OP0232 FEMALE VERSUS MALE BURDEN OF PSORIATIC ARTHRITIS IS HIGHER AND TREATMENT PERSISTENCE SHORTER AFTER USTEKINUMAB OR TUMOUR NECROSIS FACTOR INHIBITOR TREATMENT: 1-YEAR DATA FROM THE PSABIO STUDY}

I. van der Horst-Bruinsma ${ }^{1}$, M. Nurmohamed ${ }^{2}$, A. Van Kuijk ${ }^{2}$, S. Siebert ${ }^{3}$, P. Bergmans ${ }^{4}$, K. De Vlam ${ }^{5}$, E. Gremese ${ }^{6}$, B. Joven-lbáñez ${ }^{7}$, T. Korotaeva $^{8}$, W. Noel ${ }^{9}$, P. Sfikakis ${ }^{10}$, E. Theander ${ }^{11}$, J. S. Smolen ${ }^{12}$, L. Gossec ${ }^{13,14}$. ${ }^{1}$ Amsterdam University Medical Center, Location VUmc, Department of Rheumatology and Immunology, Amsterdam, Netherlands; ${ }^{2}$ Reade and VU University Medical Center, Department of Rheumatology, Amsterdam, Netherlands; ${ }^{3}$ University of Glasgow, Institute of Infection Immunity and Inflammation, Glasgow, United Kingdom; ${ }^{4}$ Janssen-Cilag BV, Biostatistics, Breda, Netherlands; ${ }^{5}$ University Hospitals Leuven, Division of Rheumatology, Leuven, Belgium; ${ }^{6}$ Catholic University of the Sacred Heart, Fondazione Policlinico A Gemelli IRCCS, Rome, Italy; ${ }^{7}$ University Hospital 12 de Octubre, Department of Rheumatology, Madrid, Spain; ${ }^{8}$ VA Nasonova Research Institute of Rheumatology, Department of Spondyloarthritis and Psoriatic Arthritis, Moscow, Russian Federation; ${ }^{9}$ Janssen Pharmaceutica NV, Medical Affairs, Beerse, Belgium; ${ }^{10}$ National and Kapodistrian University of Athens Medical School, First Department of Propaedeutic and Internal Medicine, Athens, Greece; ${ }^{11}$ Janssen-Cilag AB, Medical Affairs, Solna, Sweden; ${ }^{12}$ Medical University of Vienna, Division of Rheumatology, Department of Medicine III, Vienna, Austria; ${ }^{13}$ Sorbonne University, INSERM, Pierre Louis Institute of Epidemiology and Public Health, Paris, France; ${ }^{14}$ Pitié-Salpêtrière Hospital, AP-HP, Department of Rheumatology, Paris, France

Background: Sex-related differences in biologic treatment of psoriatic arthritis (PsA) have been insufficiently studied in a real-world setting.

Objectives: To evaluate impact of sex on PsA, treatment effectiveness and persistence after 1 year of biologic treatment.

Methods: PsABio (NCT02627768) is a multinational, prospective real-world study in PsA with ustekinumab (UST) or TNF inhibitor (TNFi) as 1st/2nd/3rd-line biologic. Males and females were compared for disease activity and patient-reported outcomes. Descriptive statistics including $95 \% \mathrm{Cl}$ at baseline (BL) and 12 $( \pm 3$ ) months (LOCF) follow-up are presented. Intra-sex comparisons between UST and TNFi cohorts were done by logistic regression analysis, with propensity score adjustment for imbalanced BL covariates and non-response imputation for stopping/switching biologic drugs.

Results: Among 494 females and 399 males, age and disease duration were similar. However, differences in disease characteristics at BL were considerable:

Table 1. Patient and disease characteristics at $B L$ and 1-year by sex

\begin{tabular}{|c|c|c|c|c|}
\hline & BL female & BL male & $\begin{array}{l}\text { 1-year LOCF } \\
\text { female }\end{array}$ & $\begin{array}{c}\text { 1-year LOCF } \\
\text { male }\end{array}$ \\
\hline \multicolumn{5}{|l|}{ Biologic line, \% } \\
\hline $1^{\text {st }}$ & 46 & 55 & N/A & $\mathrm{N} / \mathrm{A}$ \\
\hline $2^{\text {nd }}$ & 34 & 33 & & \\
\hline $3^{\text {rd }}$ & 20 & 13 & & \\
\hline \multicolumn{5}{|l|}{ Co-treatment, \% } \\
\hline MTX & 37.4 & 34.3 & N/A & $\mathrm{N} / \mathrm{A}$ \\
\hline Corticosteroids & 34.6 & 32.1 & & \\
\hline NSAIDs & 59.9 & 64.4 & & \\
\hline Antidepressant & 7.9 & 2.5 & & \\
\hline Comorbidities, \% & & & N/A & $\mathrm{N} / \mathrm{A}$ \\
\hline Cardiovascular & 69.0 & 59.4 & & \\
\hline metabolic syndrome & 40.1 & 31.7 & & \\
\hline Obesity & 35.2 & 23.7 & & \\
\hline Anxiety/depression & 12.6 & 7.5 & & \\
\hline Smoking status, \% & & & N/A & $\mathrm{N} / \mathrm{A}$ \\
\hline Never & 54.9 & 41.9 & & \\
\hline Past & 16.8 & 26.6 & & \\
\hline Current & 22.7 & 24.3 & & \\
\hline $\begin{array}{l}\text { Unknown } \\
\text { Joint counts, n }\end{array}$ & 5.7 & 7.3 & Joint counts, $n$ & \\
\hline Swollen 66 & $6.1(5.4 ; 6.9)$ & $5.6(4.7 ; 6.4)$ & $2.2(1.7 ; 2.6)$ & $1.3(1.0 ; 1.6)$ \\
\hline Tender 68 & $13.2(12.0 ; 14.4)$ & $10.0(8.9 ; 11.1)$ & $6.0(5.2 ; 6.7)$ & $3.6(2.9 ; 4.3)$ \\
\hline \multicolumn{5}{|c|}{ cDAPSA score, mean $(95 \% \mathrm{Cl})$} \\
\hline cDAPSA, $\%$ & $32.5(30.5 ; 34.4)$ & $26.9(24.9 ; 29.0)$ & $15.9(14.5 ; 17.2)$ & $10.3(9.0 ; 11.6)$ \\
\hline Remission & $1.0(0.3 ; 2.6)$ & $4.0(2.1 ; 6.7)$ & $17.8(14.1 ; 22.0)$ & $37.7(32.4 ; 43.2)$ \\
\hline Low & $6.7(4.4 ; 9.7)$ & $15.0(11.3 ; 19.4)$ & $33.0(28.3 ; 37.9)$ & $36.5(31.3 ; 42.0)$ \\
\hline Moderate & $38.9(34.0 ; 44.0)$ & $42.6(37.2 ; 48.2)$ & $34.3(29.6 ; 39.2)$ & $16.9(13.0 ; 21.4)$ \\
\hline High & $53.4(48.2 ; 58.4)$ & $38.3(33.0 ; 43.9)$ & $14.9(11.6 ; 18.9)$ & $8.9(6.0 ; 12.5)$ \\
\hline MDA & $2.3(1.0 ; 4.3)$ & $7.7(5.1 ; 11.2)$ & $27.5(23.1 ; 32.1)$ & $52.2(46.6 ; 57.7)$ \\
\hline VLDA & 0.0 & $0.9(0.2 ; 2.6)$ & $6.2(4.1 ; 9.0)$ & $19.7(15.6 ; 24.3)$ \\
\hline HAQ-DI score & $1.31(1.25 ; 1.37)$ & $0.93(0.86 ; 1.00)$ & $0.95(0.89 ; 1.02)$ & $0.53(0.47 ; 0.59)$ \\
\hline PsAID-12 score & $6.1(5.9 ; 6.3)$ & $5.1(4.9 ; 5.3)$ & $4.0(3.8 ; 4.3)$ & $2.7(2.4 ; 2.9)$ \\
\hline EQ5D VAS score & $48.6(46.6 ; 50.5)$ & $53.8(51.6 ; 55.9)$ & $59.2(56.9 ; 61.4)$ & $68.0(65.5 ; 70.4)$ \\
\hline Enthesitis & $50.7(45.9 ; 55.5)$ & $48.1(42.8 ; 53.3)$ & 32.6 (28.3; 37.3) & $18.0(14.1 ; 22.3)$ \\
\hline Dactylitis & $15.6(12.4 ; 19.3)$ & $24.7(20.4 ; 29.3)$ & $5.7(3.8 ; 8.3)$ & $4.8(2.9 ; 7.4)$ \\
\hline
\end{tabular}

Data are \% $(95 \% \mathrm{Cl})$ unless indicated otherwise. Bold data are significantly different (non-overlapping $95 \% \mathrm{Cl}$ ). females had worse scores than males for cDAPSA, HAQ-DI, EQ5D VAS, PsAID12, pain and comorbidities. At 1 year, similar improvements from $B L$ were observed between sexes, but females remained in a worse health state than males (Table). Achievement of composite endpoints MDA (including VLDA) and cDAPSA LDA (including remission) was high overall (38.6\% and $61.5 \%$, respectively), but reached by $>2$-fold and 3-fold more males than females, respectively. HAQ-DI scores remained worse for females at 1 year (0.95) than for males a BL (0.93). Enthesitis resolution was achieved in $46 \%$ of females and $75 \%$ of males. No significant differences in effectiveness of UST vs TNFi were detected between sexes (Figure). Kaplan-Meier estimated drug persistence was significantly better in males than females (log-rank $p=0.0007$ ). There was no intrasex difference between UST or TNFi in risk of stopping/switching in males or females.

Conclusion: These real-world data from PsABio on sex differences with biologic treatment suggest that females generally start biologics in a worse PsA state than males. Although treatment improvements were similar between sexes, females remained in worse health at 1 year, and stopped/ switched biologic earlier. More comprehensive treatment before severe disease manifestations evolve may improve management in females.

Acknowledgements: This study was funded by Janssen

Proportion of females and males achieving treatment targets at 1 year
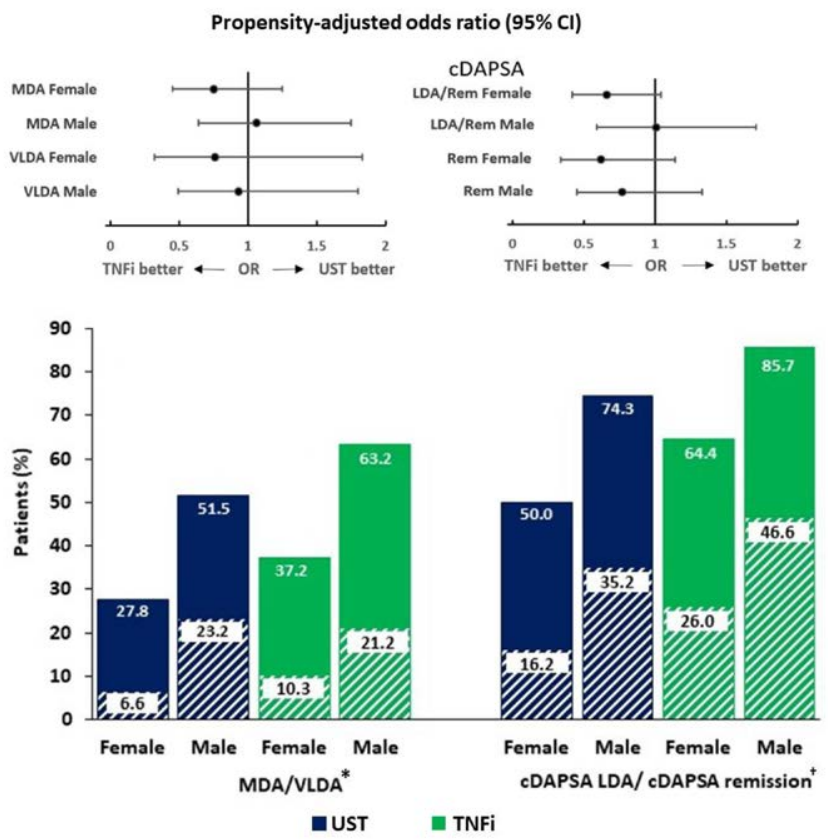

*Solid bar represents MDA and hashed bar represents VLDA.

'Solid bar represents CDAPSA LDA/remission ( $\leq 13$ ) and hashed bar represents cDAPSA remission ( $\leq 4$ ).

BL, baseline; CDAPSA, clinical Disease Activity in Psoriatic Arthritis; Cl, confidence interval; EQ5D, EuroQoL 5-dimensional questionnaire; HAQ-DI, Health Assessment Questionnaire-Disability Index; LOCF, last observation carried forward; LDA, low disease activity; MDA, minimal disease activity; MTX methotrexate; N/A, not available; NSAID, non-steroidal anti-inflammatory drugs; OR, odds ratio; PsAID12, Psoriatic Arthritis Impact of Disease 12-item questionnaire; Rem, remission; TNFi, tumour necrosis factor inhibitor; UST, ustekinumab; VAS, visual analogue scale; VLDA, very low disease activity

Disclosure of Interests: Irene van der Horst-Bruinsma Consultant of: AbbVie, Lilly, MSD, Novartis, UCB, Grant/research support from: AbbVie, MSD Pfizer, UCB, Michael Nurmohamed Speakers bureau: AbbVie, Bristol-Myers Squibb, Celgene, Eli Lilly, Janssen, Menarini, MSD, Mundipharma, Pfizer, Roche, Sanofi, UCB, Consultant of: AbbVie, Bristol-Myers Squibb, Celgene, Eli Lilly, Galapagos, Janssen, Menarini, MSD, Mundipharma, Pfizer, Roche, Sanofi, UCB, Grant/research support from: AbbVie, Bristol-Myers Squibb, Celgene, Eli Lilly, Galapagos, Gilead, Janssen, Menarini, MSD, Mundipharma, Pfizer, Roche, Sanofi, UCB, Arno Van Kuijk Consultant of: AbbVie, Janssen, LEO Pharma, Novartis, Grant/research support from: Janssen, Stefan Siebert Speakers bureau: AbbVie, Amgen (previously Celgene), Biogen, Janssen, Novartis, UCB, Consultant of: AbbVie, Janssen, UCB, Grant/ research support from: Amgen (previously Celgene), Boehringer Ingelheim, Bristol-Myers Squibb, GSK, Janssen, Novartis, Pfizer, UCB, Paul Bergmans Shareholder of: Johnson \& Johnson, Employee of: Janssen, Kurt de Vlam Speakers bureau: AbbVie, Amgen, Eli Lilly, Novartis, UCB, Paid instructor 
for: Amgen, Galapagos, UCB, Consultant of: Eli Lilly, Johnson \&Johnson, Novartis Galapagos, UCB, Grant/research support from: Celgene, Elisa Gremese: None declared, Beatriz Joven-Ibáñez Speakers bureau: AbbVie, Celgene, Janssen, MSD, Novartis, Pfizer, Tatiana Korotaeva Speakers bureau: AbbVie, Amgen, Biocad, Janssen, Lilly, MSD, Novartis, Novartis-Sandoz, Pfizer, UCB, Consultant of: AbbVie, Amgen, Biocad, Janssen, Lilly, MSD, Novartis, Novartis-Sandoz, Pfizer, UCB, Grant/research support from: Pfizer, Wim Noel Employee of: Janssen, Petros Sfikakis Consultant of: AbbVie, Actelion, Boehringer Ingelheim, Enorasis, Farmaserv-Lilly, Genesis, Gilead, MSD, Novartis, Pfizer, UCB, Grant/research support from: AbbVie, Amgen, Boehringer Ingelheim, Faran, Janssen, Pfizer, Roche, Elke Theander Employee of: Janssen, Josef S. Smolen Speakers bureau: AbbVie, Amgen, AstraZeneca, Astro, Bristol-Myers Squibb, Celgene, Celltrion, Chugai, Gilead, ILTOO, Janssen, Lilly, MSD, Novartis-Sandoz, Pfizer, Roche, Samsung, Sanofi, UCB, Consultant of: AbbVie, AstraZeneca, Lilly, Novartis, Roche, Laure Gossec Consultant of: AbbVie, Amgen, Bristol-Myers Squibb, Biogen, Celgene, Galapagos, Gilead, Janssen, Lilly, Novartis, Pfizer, Samsung Bioepis, Sanofi-Aventis, UCB, Grant/research support from: Amgen, Galapagos, Janssen, Lilly, Pfizer, Sandoz, Sanofi

DOI: 10.1136/annrheumdis-2021-eular.1575

\section{OP0233 EFFICACY AND SAFETY OF UPADACITINIB IN PATIENTS WITH PSORIATIC ARTHRITIS AND AXIAL INVOLVEMENT}

A. Deodhar ${ }^{1}$, R. Ranza ${ }^{2}$, F. Ganz ${ }^{3}$, T. Gao ${ }^{4}$, J. Anderson ${ }^{4}$, A. Ostor ${ }^{5}$. ${ }^{1}$ Oregon Health \& Science University, Rheumatology, Portland, United States of America; ${ }^{2}$ Universidade Federal de Uberlândia, Rheumatology, Uberlândia, Brazil; ${ }^{3}$ AbbVie Inc., Rheumatology, Baar, Switzerland; ${ }^{4}$ AbbVie Inc., Rheumatology, North Chicago, United States of America; ${ }^{5}$ Cabrini Hospital, Monash University \& Emeritus Research, Melbourne, Australia

Background: Patients (pts) with psoriatic arthritis (PsA) and axial involvment exhibit greater disease activity and quality of life impairments compared with those without axial involvment

Objectives: To characterize PsA pts with and without axial involvement and compare efficacy of UPA vs placebo (PBO) in PsA pts with axial involvement.

Methods: In SELECT-PsA 1 (NCT03104400; N=1705, non-biologic DMARD IR) and SELECT-PsA 2 (NCT03104374; N=642, biologic DMARD IR), pts with active PsA ( $\geq 3$ swollen and $\geq 3$ tender joints), active or historical psoriasis, and on $\leq 2$ non-biologic DMARDs were randomized to once daily UPA $15 \mathrm{mg}$, UPA $30 \mathrm{mg}$, adalimumab $40 \mathrm{mg}$ every other week (SELECT-PsA 1 only), or PBO. Efficacy was assessed in pts with axial involvement (diagnosed by investigators based on totality of information) pooled from the 2 studies. Assessments included change from BL in BASDAI, BASDAI Q2 (neck/back/hip pain) and Q3 (joint swelling/pain), and the AS Disease Activity Score (ASDAS-CRP), and percentage with BASDAI 50 response, ASDAS inactive disease (ID), ASDAS low disease activity (LDA), ASDAS major improvement (MI), and ASDAS clinically important improvement (CII). Uveitis and inflammatory bowel disease (IBD) adverse events were reviewed. Data on 24-week PBO-controlled period are presented.

Results: Prevalence of axial involvment was $31.3 \%$ in SELECT-PsA 1 and $34.2 \%$ in SELECT-PsA 2 (Table). Treatment with UPA $15 \mathrm{mg}$ and $30 \mathrm{mg}$ resulted in significantly greater improvements from BL in the BASDAI, BASDAI Q2 (neck/back/ hip pain) and Q3 (joint swelling/pain) and ASDAS-CRP at weeks 12 and 24 vs PBO (Figure). Similarly, significantly higher percentages of pts on UPA $15 \mathrm{mg}$ and $30 \mathrm{mg}$ achieved BASDAI 50, ASDAS ID, LDA, MI, and Cll at weeks 12 and 24 vs PBO (Figure). One pt on UPA $30 \mathrm{mg}$ had incident uveitis, and no IBD was reported on UPA.

Conclusion: PsA pts with axial involvement had higher BL disease burden compared with those without axial involvement. UPA was efficacious in treating axial symptoms in pts with psoriatic spondylits.

REFERENCES:

[1] van der Heijde D, et al. Lancet. 2019;394(10214):2108-2117.

Acknowledgements: Abbvie funded the study. AbbVie participated in study design, research, analysis, data collection, interpretation of the data, reviewing, and approval. All authors had access to the relevant data and participated in the drafting, review, and approval of the abstract. No honoraria or payments were made for authorship. Medical writing support was provided by M Mehta, and J Matsuura of ICON plc (North Wales, PA) and was funded by AbbVie.
Table 1. Demographics and Baseline Characteristics

\begin{tabular}{|c|c|c|c|c|c|c|}
\hline \multirow[b]{2}{*}{$\begin{array}{l}\text { Parameter, } \\
\text { mean (SD) }\end{array}$} & \multicolumn{3}{|c|}{ SELECT-PsA 1} & \multicolumn{3}{|c|}{ SELECT-PsA 2} \\
\hline & $\begin{array}{c}\text { With } \\
\text { Psoriatic } \\
\text { Spondylitis } \\
(n=534)\end{array}$ & $\begin{array}{l}\text { Without } \\
\text { Psoriatic } \\
\text { Spondylitis } \\
(n=1170)\end{array}$ & $\begin{array}{c}P \\
\text { value }^{*}\end{array}$ & $\begin{array}{c}\text { With } \\
\text { Psoriatic } \\
\text { Spondylitis } \\
(n=219)\end{array}$ & $\begin{array}{l}\text { Without } \\
\text { Psoriatic } \\
\text { Spondylitis } \\
(n=421)\end{array}$ & $\begin{array}{c}P \\
\text { value }^{*}\end{array}$ \\
\hline BMI $\left(\mathrm{kg} / \mathrm{m}^{2}\right)$ & $29.9(6.5)$ & $30.5(6.9)$ & .0810 & $31.6(8.0)$ & $31.3(6.9)$ & .6226 \\
\hline TJC68 & $21.6(15.1)$ & $19.2(13.5)$ & .0022 & $27.5(18.0)$ & $23.3(16.2)$ & .0027 \\
\hline SJC66 & $11.7(9.4)$ & $11.0(7.9)$ & .1184 & $12.9(9.2)$ & $11.7(8.7)$ & .0804 \\
\hline $\begin{array}{l}\text { Physician's Global } \\
\text { Assessment } \\
\text { (NRS 0-10) }\end{array}$ & $6.7(1.6)$ & $6.5(1.7)$ & .0437 & $6.6(1.8)$ & $6.5(1.7)$ & .1897 \\
\hline HAQ-DI & $\begin{array}{c}1.2(0.6) \\
n=531\end{array}$ & $\begin{array}{l}1.1(0.6) \\
n=1164\end{array}$ & .0170 & $\begin{array}{c}1.2(0.6) \\
n=218\end{array}$ & $\begin{array}{c}1.2(0.7) \\
n=416\end{array}$ & .2049 \\
\hline $\begin{array}{l}\text { Presence of } \\
\text { dactylitis, } \\
\mathrm{n}(\%)^{\dagger}\end{array}$ & $188(35.2)$ & $328(28.0)$ & .0028 & $69(31.5)$ & $100(23.8)$ & .0348 \\
\hline $\begin{array}{l}\text { Presence of } \\
\text { enthesitis, } \\
\mathrm{n}(\%)^{\ddagger}\end{array}$ & $432(80.9)$ & $884(75.6)$ & .0147 & $189(86.3)$ & $337(80.0)$ & .0125 \\
\hline ASDAS-CRP & $\begin{array}{c}3.4(0.9) \\
n=530\end{array}$ & $\begin{array}{l}3.1(1.0) \\
n=1161\end{array}$ & $<.0001$ & $\begin{array}{c}3.3(1.0) \\
n=217\end{array}$ & $\begin{array}{c}3.2(1.1) \\
n=416\end{array}$ & .1032 \\
\hline BASDAI & $\begin{array}{c}5.8(2.0) \\
n=530\end{array}$ & $\begin{array}{c}5.3(2.2) \\
n=1161\end{array}$ & $<.0001$ & $\begin{array}{c}6.2(2.2) \\
n=217\end{array}$ & $\begin{array}{c}5.8(2.2) \\
n=416\end{array}$ & .0673 \\
\hline $\begin{array}{l}\text { Morning Stiffness } \\
\text { Duration (NRS } \\
0-10 ; \\
\text { BASDAI Q6) }\end{array}$ & $5.0(3.0)$ & $4.7(3.0)$ & .0368 & $5.6(3.2)$ & $5.1(3.0)$ & .0454 \\
\hline $\begin{array}{l}\text { Patient's } \\
\text { Assessment of } \\
\text { Inflammatory } \\
\text { Neck, Back, or Hip } \\
\text { Pain (NRS 0-10; } \\
\text { BASDAI Q2) }\end{array}$ & $\begin{array}{c}n=530 \\
5.8(2.7)\end{array}$ & $\begin{array}{c}n=1161 \\
4.6(3.2)\end{array}$ & $<.0001$ & $\begin{array}{c}n=217 \\
6.4(2.8)\end{array}$ & $\begin{array}{c}n=416 \\
5.4(3.1)\end{array}$ & .0001 \\
\hline & $\mathrm{n}=530$ & $\mathrm{n}=1161$ & & $\mathrm{n}=217$ & $\mathrm{n}=416$ & \\
\hline
\end{tabular}

${ }^{*}$ Calculated by t-test for continuous variables and chi-square test for categorical values. Bolded if $<0.05$. Defined as ${ }^{\dagger} \mathrm{LDI}>0$ and ${ }^{*}$ total enthesitis count $>0$

Figure. Integrated Analysis of Efficacy in PsA Patients With Axial Involvement

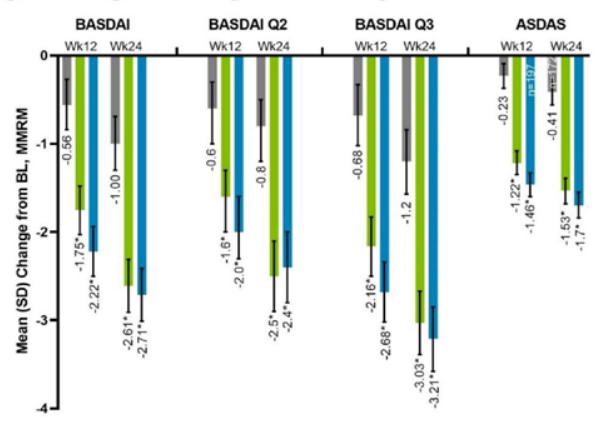

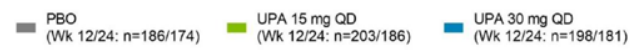

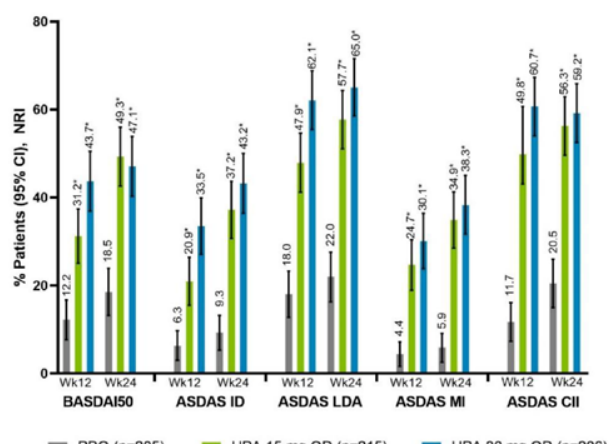

$=$ PBO (n=205) $=$ UPA $15 \mathrm{mg}$ QD (n=215) UPA $30 \mathrm{mg}$ QD (n=206)

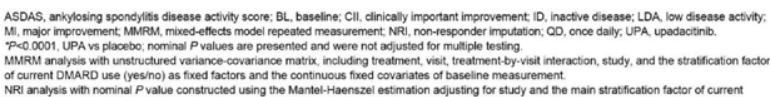

